\title{
Original
}

\section{Usefulness of Continuous Regional Arterial Infusion with Doripenem and Protease Inhibitors for Severe Acute Pancreatitis}

\author{
Akira YamamiYA, Katsuya KitamurA*, Yu IshiI, \\ Yoshiki Sato, Tomoyuki Iwata, Tomohiro Nomoto, \\ Tadashi Honma and Hitoshi YoshIDA
}

\begin{abstract}
Doripenem (DRPM) is a relatively new drug belonging to the carbapenem antibiotic group. We hypothesized that the pharmacological characteristics of DRPM could make it useful in the treatment of severe acute pancreatitis (SAP). We investigated the usefulness of continuous regional arterial infusion (CRAI) with DRPM and protease inhibitors for SAP. Two hundred and forty-two patients with SAP were admitted to Showa University Hospital between November 2002 and June 2013. Of these, 53 patients were treated with CRAI with carbapenem antibiotics and nafamostat mesilate (NM), a serine protease inhibitor, via the celiac and superior mesenteric arteries. Clinical outcomes were evaluated retrospectively in 34 patients treated with DRPM and 19 patients undergoing non-DRPM therapy (meropenem $\mathrm{n}=11$, imipenem $\mathrm{n}=6$; biapenem $\mathrm{n}=2$ ). The median time to commencement of oral intake was significantly shorter in the DRPM than nonDRPM group ( 9 vs 14 hospital days, respectively; $P<0.01$ ). In addition, the rate of walled-off necrosis in the DRPM group tended to be lower than in the nonDRPM group ( 37.5 vs $64.7 \%$, respectively, $P=0.069$ ). The results of the present study suggest that CRAI with DRPM and NM for SAP could have equivalent therapeutic effects to CRAI with other carbapenem antibiotics and NM.
\end{abstract}

Key words : continuous regional arterial infusion, doripenem, protease inhibitor, severe acute pancreatitis

\section{Introduction}

Severe acute pancreatitis (SAP), although a benign disease, is associated with high mortality and is often refractory to treatment. The mortality rate of SAP has been reported to be approximately $30 \%{ }^{1)}$.

Guidelines regarding pancreatitis have been amended using a revision of the Atlanta International Classification ${ }^{2)}$. In these new guidelines, the definition, severity assessment, and treatment strategies for SAP have been revised. SAP is diagnosed when there is associated organ failure that is refractory to therapy for $48 \mathrm{hr}$.

In current practice, patients are observed for 4 weeks for the development of walled-off necro-

Division of Gastroenterology, Department of Medicine, Showa University School of Medicine, 1-5-8 Hatanodai, Shinagawa-ku, Tokyo 142-8666, Japan.

* To whom corresponding should be addressed. 
sis (WON), a complication of infectious pancreatitis, which, if it occurs, is then treated by the recommended therapeutic approach of necrosectomy using a low invasive approach ${ }^{3)}$. Infectious pancreatic complications are an important prognostic factor for SAP; it is reported that continuous regional arterial infusion (CRAI) with antibiotics and protease inhibitors is effective in preventing infectious pancreatic complications and reducing mortality following SAP ${ }^{4,5)}$. Recently, studies have been published describing CRAI with carbapenem antibiotics ${ }^{6-11)}$. Based on the results of these studies, we adopted CRAI with carbapenem antibiotics and protease inhibitors for the treatment of SAP. SAP is associated with a high mortality rate. Therefore, it is very difficult to perform a prospective study for SAP.

The aim of the present study was to evaluate the usefulness of CRAI with doripenem (DRPM) and the serine protease inhibitor nafamostat mesilate (NM) for SAP. DRPM is a relatively new drug belonging to the carbapenem antibiotic group. We hypothesized that the pharmacological characteristics of DRPM could make it useful in the treatment of SAP. We compared the safety and efficacy of CRAI with DRPM and NM for SAP with non-DRPM therapy (i.e. treatment with other carbapenem antibiotics) by assessing adverse events and the mortality of SAP following treatment.

\section{Material and methods}

Two hundred and forty-two patients with SAP were admitted to Showa University Hospital between November 2002 and June 2013. Of these, 53 patients underwent treatment with CRAI with carbapenem antibiotics and NM via the celiac and superior mesenteric arteries. Clinical outcomes, such as infectious complications and mortality, were reviewed retrospectively and compared between the 34 patients who received DRPM and the 19 patients who were treated with non-DRPM therapy (meropenem $[\mathrm{MEPM}] \mathrm{n}=11$; imipenem $[\mathrm{IPM}] \mathrm{n}=6]$; biapenem [BIPM] $\mathrm{n}=2$ ).

In Japan, the prognostic factors and computed tomography (CT) grading for acute pancreatitis ${ }^{12)}$, developed by the Research Committee of Intractable Diseases of the Pancreas (Ministry of Health, Labour and Welfare), are used for the assessment of the severity of acute pancreatitis (Table 1).

CRAI was used in patients with severe acute necrotizing pancreatitis, defined as a lack of enhancement of pancreatic parenchyma on contrast-enhanced (CE) CT. The drugs administered by CRAI were NM ( $240 \mathrm{mg} /$ day) together with either $1.5 \mathrm{~g} /$ day DRPM $(0.5 \mathrm{~g}$ every $8 \mathrm{hr})$, $2 \mathrm{~g} /$ day MEPM $(1 \mathrm{~g}$ every $12 \mathrm{hr}), 2 \mathrm{~g} /$ day IPM $(1 \mathrm{~g}$ every $12 \mathrm{hr})$, or $1.2 \mathrm{~g} /$ day BIPM $(0.6 \mathrm{~g}$ every $12 \mathrm{hr}$ ). The antibiotic used was chosen at the discretion of the attending physician.

Continuous variables are expressed as median values with the range in parentheses. Statistical analyses were performed using StatMate III software (ATMS, Tokyo, Japan). Data were analyzed by the Mann-Whitney $U$-test and Chi-squared test, with $P<0.05$ considered significant.

The present retrospective study was approved by the Ethics Committee of Showa University Hospital. 
Table 1. Japanese severity scoring system for acute pancreatitis (Ministry of Health, Labour and Welfare of Japan, 2008 revision)

Prognostic factors (1 point for each factor)

1. Base excess $\leq-3 \mathrm{mEq} / 1$ or shock (systolic blood pressure $<80 \mathrm{mmHg}$ )

2. $P_{\mathrm{a}} \mathrm{O}_{2} \leq 60 \mathrm{mmHg}$ (room air) or respiratory failure (requiring ventilatory management)

3. $\mathrm{BUN} \geq 40 \mathrm{mg} / \mathrm{dl}$ or creatinine $\geq 2.0 \mathrm{mg} / \mathrm{dl}$ or oliguria (daily urine output $<400 \mathrm{ml}$ even after intravenous fluid resuscitation)

4. Lactate dehydrogenase $\geq 2$ ULN

5. Platelet count $\leq 100,000 / \mathrm{mm}^{3}$

6. Serum $\mathrm{Ca} \leq 7.5 \mathrm{mg} / \mathrm{dl}$

7. C-Reactive protein $\geq 15 \mathrm{mg} / \mathrm{dl}$

8. No. positive measures in SIRS criteria $\geq 3$

9. Age $\geq 70$ years

CT grade based on contrast-enhanced CT

1 Extrapancreatic progression of inflammation

Anterior pararenal space

Root of mesocolon

Beyond lower pole of kidney

0 points

1 point

2 points

2. Hypo-enhanced lesion of the pancreas

The pancreas is conveniently divided into three segments (head, body, and tail) Localized in each segment or only surrounding the pancreas 0 points Extends to two segments Occupies entire two segments or more

1 point $1+2=$ total score

Total score $=0$ or 1

2 points

Total score $=2$

Total score $\geq 3$

Assessment of severity

If the prognostic factors score is $\geq 3$ or CT grade is $\geq 2$, the disease is graded as 'severe'

The systemic inflammatory response syndrome (SIRS) criteria include body temperature $>38^{\circ} \mathrm{C}$ or $<36^{\circ} \mathrm{C}$, heart rate $>90$ b.p.m., respiratory rate $>20$ breaths $/ \mathrm{min}$ or $P_{\mathrm{a}} \mathrm{CO}_{2} \leq 32$ torr, and white blood cell counts $>12,000$ cells $/ \mathrm{mm}^{3},<4000$ cells $/ \mathrm{mm}^{3}$, or $>10 \%$ immature (band) forms.

BUN, blood urea nitrogen; ULN, upper limit of normal ; CT, computed tomography.

\section{Results}

The median age of the 53 SAP patients (41 men, 12 women) was 49 years (range $15 \sim 85$ years). Disease etiology included alcoholic pancreatitis in 34 patients, gallstones in two, idiopathic pancreatitis in 13 , and other diseases in the remaining four patients. The median Japanese prognostic factors score within $48 \mathrm{hr}$ of admission was 4 points (range $0 \sim 8$ points). CE-CT Grade 2 disease was present in 30 patients, whereas Grade 3 disease was present in 23 patients. The median Acute Physiology and Chronic Health Evaluation (APACHE) II score was 10 points (range $0 \sim 31$ points), and the median sequential organ failure assessment (SOFA) score was 2 points (range $0 \sim 10$ points). The median day of commencement of CRAI was Hospital Day 1 (range Days $1 \sim 4$ ), the median duration of CRAI was 5 days (range $3 \sim 7$ days), and the mortality rate was $9.4 \%$.

There were no significant differences in patient characteristics between the DRPM and nonDRPM groups in terms of age, sex, etiology, Japanese prognostic factors score, CE-CT grade, APACHE-II score, SOFA score, starting day of CRAI, and duration of CRAI (Table 2). No 
Table 2. Patient characteristics in doripenem (DRPM)-treated and -untreated (non-DRPM) patients

\begin{tabular}{lccc}
\hline & DRPM & Non-DRPM \\
& group $(\mathrm{n}=34)$ & $P$ value \\
& $51(19 \sim 85)$ & $48(15 \sim 83)$ & $\mathrm{NS}$ \\
\hline Age (years) & $27 / 7$ & $14 / 5$ & $\mathrm{NS}$ \\
No. men / women & $24 / 1 / 7 / 2$ & $10 / 1 / 6 / 2$ & $\mathrm{NS}$ \\
Etiology (alcohol / gallstones / idiopathic/ others) & $3(0 \sim 7)$ & $4(1 \sim 8)$ & $\mathrm{NS}$ \\
Japanese prognostic factors score (points) & $0 / 22 / 12$ & $0 / 8 / 11$ & $\mathrm{NS}$ \\
\hline CE-CT grade (1/2/3) & $11(0 \sim 28)$ & $9(1 \sim 31)$ & $\mathrm{NS}$ \\
APACHE-II score (points) & $3(0 \sim 10)$ & $2(0 \sim 10)$ & $\mathrm{NS}$ \\
SOFA score (points) & $1(1 \sim 3)$ & $1(1 \sim 4)$ & $\mathrm{NS}$ \\
Start time of CRAI (hospital day) & $5(3 \sim 5)$ & $5(3 \sim 7)$ & $\mathrm{NS}$
\end{tabular}

Data are presented as median values with the range in parentheses, or as the number of patients in each group. CE-CT, contrast-enhanced computed tomography; APACHE, Acute Physiology and Chronic Health Evaluation; SOFA, Sequential Organ Failure Assessment; CRAI, continuous regional arterial infusion.

significant differences were observed between the two groups with regard to the disappearance of abdominal pain, duration of administration of antibiotics, early organ dysfunction rate, late severe infection rate, surgery rate, duration of admission, and mortality. The median day of commencement of oral feeding was significantly earlier in the DRPM than non-DRPM group (9 vs 14 hospital days, respectively; $P<0.01)$. The rate of WON tended to be lower in the DRPM than non-DRPM group $(37.5 \%$ vs $64.7 \%$, respectively; $P=0.069$; Table 3$)$. Adverse events due to CRAI with DRPM and NM for SAP included increased serum bilirubin levels in five patients (two patients had increased aspartate aminotransferase and alanine aminotransferase levels), one case of subarachnoid hemorrhage $(\mathrm{SAH})$, and one case of obstruction of the artery of the lower extremity.

\section{Discussion}

When acute pancreatitis is suspected, its severity needs to be determined so that appropriate treatment can be administered. In severe cases, strict circulatory and respiratory management are necessary.

In Showa University Hospital, CRAI is performed in patients with severe acute necrotizing pancreatitis, and continuous hemodiafiltration (CHDF) is performed in patients with SAP with organ dysfunction. Further, we provide early enteral nutrition as infection prophylaxis for SAP.

In Japan, it is not considered necessary to administer antibiotics prophylactically to patients with mild acute pancreatitis because of the low rate of infectious complications and mortality. Although prophylactic administration of antibiotics for SAP can improve the prognosis and decrease the incidence of infectious pancreatic complications, it is necessary to consider the antibacterial spectrum, pancreatic tissue concentration, and dosing period of antibiotics for SAP. 
Table 3. Clinical outcomes in doripenem (DRPM)-treated and -untreated (non-DRPM) patients

\begin{tabular}{lccc}
\hline & $\begin{array}{c}\text { DRPM } \\
\text { group }(\mathrm{n}=34)\end{array}$ & $\begin{array}{c}\text { Non-DRPM } \\
\text { group (n=19) }\end{array}$ & $P$ value \\
\hline Disappearance of abdominal pain (hospital day) & $5(2 \sim 12)$ & $5(3 \sim 15)$ & NS \\
Start day of oral intake (hospital day) & $9(4 \sim 35)$ & $14(10 \sim 93)$ & $<0.01$ \\
Duration of administration of antibiotics (days) & $12(3 \sim 57)$ & $13(3 \sim 143)$ & NS \\
Early organ dysfunction rate & $64.7 \%(22 / 34)$ & $84.2 \%(16 / 19)$ & NS \\
\hline Late severe infection rate & $13.3 \%(4 / 30)$ & $17.6 \%(3 / 17)$ & NS \\
Surgery rate & $2.9 \%(1 / 34)$ & $5.3 \%(1 / 19)$ & NS (0.069) \\
WON rate & $37.5 \%(12 / 32)$ & $64.7 \%(11 / 17)$ & $25(3 \sim 172)$ \\
Duration of admission (days) & $21(3 \sim 115)$ & $10.5 \%(2 / 19)$ & NS \\
Mortality rate & $8.8 \%(3 / 34)$ & NS
\end{tabular}

Data are given as median values with the range in parentheses, or as percentages with the number of patients / total number of patients in parentheses.

WON, walled-off necrosis.

Interestingly however, one published study in 2006 does not recommend prophylactic antibiotic therapy in patients with $\mathrm{SAP}^{13)}$.

Buchler et $a l^{14)}$ examined pancreatic tissue concentrations of different antibiotics and reported that imipenem (carbapenem antibiotics), ofloxacin, and ciprofloxacin (new quinolone) had high pancreatic tissue concentrations and extensive bactericidal activity against most bacteria. Therefore, we examined the usefulness of prophylactic antibiotics in SAP in the present study. There have been some reports published regarding the prophylactic use of antibiotics for severe pancreatitis ${ }^{15-26)}$. The efficacy of prophylactic antibiotics for acute pancreatitis was first reported after the introduction of carbapenem antibiotics in the 1990s, including a report regarding the use of imipenem by Pederzoli et $a l^{15)}$. Recently, carbapenem antibiotics for SAP have been recommended in $\operatorname{Japan}^{7-10)}$.

Some placebo-controlled double-blind studies have evaluated transvenous prophylactic antibiotic therapy ${ }^{21,22)}$. These studies suggest that prophylactic antibiotic therapy for SAP does not necessarily lead to decreases in infectious pancreatic complications and mortality rate. However, these studies varied in the type of antimicrobial agent used, the route of administration, and the day on which antibiotics were started. Therefore, denying early prophylactic antibiotic therapy based on these studies is not appropriate.

Conversely, a randomized controlled trial (RCT) on early prophylactic antibiotic therapy for SAP reported decreases in infectious pancreatic complications and mortality rate with such therapy ${ }^{25)}$. Manes et $a l^{22)}$ conducted an RCT using MEPM for necrotizing pancreatitis. They divided patients into two groups based on the timing of commencement of antibiotic administration (i.e. at admission and after CE-CT confirmation of the pancreatic necrotic area). Pancreatic infection occurred in $13 \%$ of patients in the former group, compared with $31 \%$ of patients in 
the latter group, with extrapancreatic infection observed in $17 \%$ and $45 \%$ of patients, respectively. Pancreatic and extrapancreatic infection rates in the former group were significantly lower than in the latter group. In addition, the former group had lower surgery rates and a shorter period of admission than patients in the latter group. Therefore, the results of the study of Manes et $\mathrm{al}^{22)}$ suggest that the time of initiation of antibiotic therapy is an important determinant of patient outcome.

CRAI for acute necrotizing pancreatitis was reported by Takeda et $a l^{4)}$, and came to be recognized as appropriate therapy for SAP. The purpose of CRAI is to directly deliver a high concentration of the protease inhibitor and antibiotic to the site of inflammation and necrotic tissue by intra-arterial injection to prevent local pancreatic inflammation and infection. NM acts as a protease inhibitor at low concentrations and as an anticoagulant at high concentrations. It is estimated that local pancreatic concentrations of drugs administered by intra-arterial injection for CRAI can reach levels nine fold higher, or more, than those seen after intravenous infusion ${ }^{27)}$. Takeda $e a^{4)}$ reported that NM inhibits the extension of pancreatic necrosis by its anticoagulant action in the microcirculation, and is effective for the prevention of vasospasm and vascular occlusion. In that study, intraperitoneal infections, infections due to pancreatic necrosis, and mortality were significantly lower in the NM than non-NM group.

Hayashi $e t a l^{6)}$ have reported the usefulness of CRAI via the celiac artery, whereas Takagi and Isaji ${ }^{5)}$ have reported the usefulness of CRAI via the superior mesenteric artery.

In studies that divided patients with SAP into CRAI and non-CRAI groups, infectious pancreatic complications, surgical rate, and mortality were lower in the CRAI than non-CRAI group ${ }^{7,8)}$. It has also been reported that patients who received CRAI for SAP with gabexate mesilate as the protease inhibitor had a shorter duration until the disappearance of abdominal pain, lower systemic inflammatory response syndrome (SIRS) positive scores, and a shorter duration of hospitalization than patients in the non-CRAI group ${ }^{9)}$.

Ishikawa et $a l^{10)}$ reported on the usefulness of CRAI with NM and IPM for SAP. The mortality rate was significantly lower in the group that started CRAI within 3 days of the onset of SAP compared with the group that started CRAI after 3 days after onset (0\% vs 66.7\%, respectively). Ishikawa et $a l^{10)}$ concluded that the timing of CRAI initiation is extremely important, and that CRAI should be initiated within 3 days of the onset of SAP for greater efficacy.

An RCT of CRAI for SAP was first reported by Piascik et al ${ }^{11)}$. In that study, 78 patients were randomly divided within $72 \mathrm{hr}$ from the onset of acute pancreatitis and hospitalization into two groups $(\mathrm{n}=39$ in each) : a CRAI group and a non-CRAI group. The CRAI group was treated continuously with $240 \mathrm{mg} /$ day NM and $1 \mathrm{~g} /$ day IPM for 5 days as an arterial infusion via one of the arteries perfusing the pancreas, following which IPM was given intravenously $(0.5 \mathrm{~g}$ every $8 \mathrm{hr})$ for at least 9 days. The non-CRAI group received IPM $(0.5 \mathrm{~g}$ every $8 \mathrm{hr})$ intravenously for 14 days. The mortality rate was lower in the CRAI than non-CRAI group, indicating that CRAI for SAP with a protease inhibitor and antibiotic is effective in preventing adverse events and reducing mortality rates.

Recently, a pharmacokinetic-pharmacodynamic theory was proposed ${ }^{28,29)} . \quad \beta$-Lactam antibiotics, 
such as carbapenem antibiotics, are drugs with time-dependent bactericidal activity. In vivo, the proliferative inhibitory effect and maximal bactericidal action correlate with the time for which the drug concentration exceeds its minimum inhibitory concentration ( $\mathrm{MIC} ; \% \mathrm{~T}>\mathrm{MIC}$ ).

In the present study, we chose DRPM as the antibiotic to be evaluated for the following reasons: (1) DRPM is highly effective against aerobic Gram-negative and Gram-positive bacteria, as well as anaerobic bacteria, and it suppresses the growth of antimicrobial-resistant Pseudomonas aeruginosa; (2) DRPM, MEPM, and IPM exhibit potent activity, with an $\mathrm{MIC}_{90}$ of 4, 16, and $32 \mu \mathrm{g} / \mathrm{ml}$, respectively, and DRPM is clinically effective against $P$. aeruginosa infection ; (3) DRPM does not show any cross-resistance with MEPM and IPM against P. aeruginosa; (4) incompatibility between DRPM and protease inhibitors is low; and (5) DRPM is a drug with little effect on the central nervous system ${ }^{30-36)}$.

In addition, on the basis of changes in plasma concentrations in healthy adults in a Phase I study ${ }^{14)}$, it has been suggested that as the number of doses of DRPM increases, the time above the MIC of DRPM increases. In the present study, the incidence of WON in the DRPM group tended to be lower than in the non-DRPM group, indicating the clinical efficacy of CRAI with DRPM and NM. One study compared the $\mathrm{MIC}_{90}$ of DRPM, IPM, and MEPM for the main microorganism strains affected by them. In most strains, the $\mathrm{MIC}_{90}$ of DRPM was lower than that of IPM and MEPM ${ }^{29)}$.

In the present study, it is possible that synergistic actions between DRPM and NM led to the observed decrease in the incidence of WON.

In conclusion, the results of the present study suggest that CRAI with DRPM and NM for SAP could provide equivalent therapeutic effects to those seen following CRAI with other carbapenem antibiotics and NM.

\section{Conflict of interest disclosure}

The authors have no sources of funding or conflicts of interest to declare.

\section{References}

1) Petrov MS, Shanbhag S, Chakraborty M, et al. Organ failure and infection of pancreatic necrosis as determinants of mortality in patients with acute pancreatitis. Gastroenterology. 2010;139:813-820.

2) Banks PA, Bollen TL, Dervenis C, et al. Classification of acute pancreatitis-2012: revision of the Atlanta classification and definitions by international consensus. Gut. 2013;62:102-111.

3) Trikudanathan G, Arain M, Attam R, et al. Interventions for necrotizing pancreatitis: an overview of current approaches. Expert Rev Gastroenterol Hepatol. 2013;7:463-475.

4) Takeda K, Matsuno S, Sunamura M, et al. Continuous regional arterial infusion of protease inhibitor and antibiotics in acute necrotizing pancreatitis. Am J Surg. 1996;171:394-398.

5) Takagi K, Isaji S. Therapeutic efficacy of continuous arterial infusion of an antibiotic and a protease inhibitor via the superior mesenteric artery for acute pancreatitis in an animal model. Pancreas. 2000;21:279-289.

6) Hayashi J, Kawarada Y, Isaji S, et al. Therapeutic effects of continuous intraarterial antibiotic infusion in preventing pancreatic infection in experimental acute necrotizing pancreatitis. Pancreas. 1996;13:184-192.

7) Imaizumi H, Kida M, Nishimaki $\mathrm{H}$, et al. Efficacy of continuous regional arterial infusion of a protease inhibitor 
and antibiotic for severe acute pancreatitis in patients admitted to an intensive care unit. Pancreas. 2004;28:369373.

8) Yasuda T, Ueda T, Takeyama Y, et al. Treatment strategy against infection: clinical outcome of continuous regional arterial infusion, enteral nutrition, and surgery in severe acute pancreatitis. $J$ Gastroenterol. 2007;42:681-689.

9) Ino Y, Arita Y, Akashi T, et al. Continuous regional arterial infusion therapy with gabexate mesilate for severe acute pancreatitis. World J Gastroenterol. 2008;14:6382-6387.

10) Ishikawa $\mathrm{T}$, Imai $\mathrm{M}$, Kamimura $\mathrm{H}$, et al. Therapeutic efficacy of continuous arterial infusion of the protease inhibitor and the antibiotics and via celiac and superior mesenteric artery for severe acute pancreatitis: pilot study. Hepatogastroenterology. 2009;56:524-528.

11) Piascik M, Rydzewska G, Milewski J, et al. The results of severe acute pancreatitis treatment with continuous regional arterial infusion of protease inhibitor and antibiotic: a randomized controlled study. Pancreas. 2010;39:863867.

12) Takeda K, Yokoe M, Takada $\mathrm{T}$, et al. Assessment of severity of acute pancreatitis according to new prognostic factors and CT grading. J Hepatobiliary Pancreat Sci. 2010;17:37-44.

13) Banks PA, Freeman ML, Practice Parameters Committee of the American College of Gastroenterology. Practice guidelines in acute pancreatitis. Am J Gastroenterol. 2006;101:2379-2400.

14) Buchler M, Malfertheiner P, Friess H, et al. Human pancreatic tissue concentration of bactericidal antibiotics. Gastroenterology. 1992;103:1902-1908.

15) Pederzoli $\mathrm{P}$, Bassi $\mathrm{C}$, Vesentini S, et al. A randomized multicenter clinical trial of antibiotic prophylaxis of septic complications in acute necrotizing pancreatitis with imipenem. Surg Gynecol Obstet. 1993;176:480-483.

16) Sainio V, Kemppainen E, Puolakkainen $\mathrm{P}$, et al. Early antibiotic treatment in acute necrotising pancreatitis. Lancet. 1995;346:663-667.

17) Delcenserie R, Yzet T, Ducroix JP. Prophylactic antibiotics in treatment of severe acute alcoholic pancreatitis. Pancreas. 1996;13:198-201.

18) Schwarz M, Isenmann $\mathrm{R}$, Meyer $\mathrm{H}$, et al. Antibiotic use in necrotizing pancreatitis. Results of a controlled study. Dtsch Med Wochenschr. 1997;122:356-361. (in German).

19) Nordback I, Sand J, Saaristo R, et al. Early treatment with antibiotics reduces the need for surgery in acute necrotizing pancreatitis: a single-center randomized study. J Gastrointest Surg. 2001;5:113-118.

20) Rokke O, Harbitz TB, Liljedal J, et al. Early treatment of severe pancreatitis with imipenem: a prospective randomized clinical trial. Scand J Gastroenterol. 2007;42:771-776.

21) Manes G, Rabitti PG, Menchise A, et al. Prophylaxis with meropenem of septic complications in acute pancreatitis: a randomized, controlled trial versus imipenem. Pancreas. 2003;27:e79-83.

22) Manes G, Uomo I, Menchise A, et al. Timing of antibiotic prophylaxis in acute pancreatitis: a controlled randomized study with meropenem. Am J Gastroenterol. 2006;101:1348-1353.

23) Isenmann R, Runzi M, Kron M, et al. Prophylactic antibiotic treatment in patients with predicted severe acute pancreatitis: a placebo-controlled, double-blind trial. Gastroenterology. 2004;126:997-1004.

24) Dellinger EP, Tellado JM, Soto NE, et al. Early antibiotic treatment for severe acute necrotizing pancreatitis: a randomized, double-blind, placebo-controlled study. Ann Surg. 2007;245:674-683.

25) Garcia-Barrasa A, Borobia FG, Pallares R, et al. A double-blind, placebo-controlled trial of ciprofloxacin prophylaxis in patients with acute necrotizing pancreatitis. J Gastrointest Surg. 2009;13:768-774.

26) Xue P, Deng LH, Zhang ZD, et al. Effect of antibiotic prophylaxis on acute necrotizing pancreatitis: results of a randomized controlled trial. J Gastroenterol Hepatol. 2009;24:736-742.

27) Mikami Y, Takeda K, Matsuda K, et al. Rat experimental model of continuous regional arterial infusion of protease inhibitor and its effects on severe acute pancreatitis. Pancreas. 2005;30:248-253.

28) Lipman J, Udy AA, Roberts JA. Do we understand the impact of altered physiology, consequent interventions 
and resultant clinical scenarios in the intensive care unit? The antibiotic story. Anaesth Intensive Care. 2011;39:9991000 .

29) Craig WA. Pharmacokinetic/pharmacodynamic parameters: rationale for antibacterial dosing of mice and men. Clin Infect Dis. 1998;26:1-10.

30) Sahm D. In vitro activity of doripenem. Clin Infect Dis. 2009;49:S11-S16.

31) Nordmann P, Picazo JJ, Mutters R, et al. Comparative activity of carbapenem testing: the COMPACT study. $J$ Antimicrob Chemother. 2011;66:1070-1078.

32) Roberts JA, Lipman J. Optimal doripenem dosing simulations in critically ill nosocomial pneumonia patients with obesity, augmented renal clearance, and decreased bacterial susceptibility. Crit Care Med. 2013;41:489-495.

33) Tazuma S, Igarashi Y, Inui K, et al. Clinical efficacy of i.v. doripenem, a new class of carbapenem, in patients with biliary tract infection: A multicenter trial. Hepatol Res. 2011;41:340-349.

34) Keam SJ. Doripenem: a review of its use in the treatment of bacterial infections. Drugs. 2008;68:2021-2057.

35) Sakyo S, Tomita H, Tanimoto $\mathrm{K}$, et al. Potency of carbapenems for the prevention of carbapenem-resistant mutants of Pseudomonas aeruginosa: the high potency of a new carbapenem doripenem. J Antibiot (Tokyo). 2006;59:220-228.

36) Tanimoto K, Tomita H, Fujimoto S, et al. Fluoroquinolone enhances the mutation frequency for meropenemselected carbapenem resistance in Pseudomonas aeruginosa, but use of the high-potency drug doripenem inhibits mutant formation. Antimicrob Agents Chemother. 2008;52:3795-3800.

[Received January 8, 2015 : Accepted January 13, 2015] 